\section{Military Technical College Kobry El-Kobbah, Cairo, Egypt.}

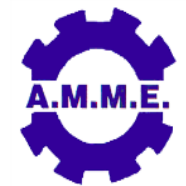
$18^{\text {th }}$ International Conference on Applied Mechanics and Mechanical Engineering.

\title{
MODELING OF THE WELD BEAD SHAPE AT DIFFERENT WELDING PARAMETERS
}

\author{
A. A. Abd Elsalam ${ }^{1}$, S. ElAtriby ${ }^{2}$, A. F. Barakat ${ }^{3}$ and M. Abdel Ghany ${ }^{4}$
}

\begin{abstract}
This paper tends to discuss the relation between welding parameters (Voltage, Arc Current and Travel Speed) and the deposited lines shape (width, height and penetration) in metal deposition process. Due to the high nonlinearity of the process ANN (artificial neural network) was found to be the best chose for representing it. ANN is trained off-line under different operating conditions then used for prediction of the system model for further optimization of the process. The results show the capability of the developed ANNs to represent the process properly. Supervised learning with back propagation technique was used to make the networks. Best network for width consisted of tansig functions with an output layer of linear function. Traingd function gave the best result for width. Best network for penetration consisted of tansig functions with an output layer of linear function. Traingd function gave the best result for penetration. Best network for height consisted of mixture of radial basis, tansig functions with an output layer of linear function. Traingd function gave the best result for height.
\end{abstract}

\section{KEYWORDS}

ANN, MIG, GMAW, Shape of weld line.

1 Assistant Lecturer at Mechanical Engineering Dept., Faculty of Engineering, Helwan University, Cairo, Egypt.

2 Lecturer at Mechanical Engineering Dept., Faculty of Engineering, Helwan University, Cairo, Egypt.

3 Professor at Mechanical Engineering Dept., Faculty of Engineering, Helwan University, Cairo, Egypt.

4 Professor at Electrical Engineering Dept., Faculty of Engineering Helwan University, Cairo, Egypt. 


\section{INTRODUCTION}

It is important to know the shape of a deposited metal line nowadays to facilitate prediction of further manufacturing processes if required. Also it is the era of 3D printing so knowing such information will serve build up and 3D techniques. MIG (metal inert gas) welding known also as GMAW (gas metal arc welding) was selected for this research because of its popularity in the industrial community, low cost, easiness of application and wide variety of wire materials.

According to previous researches [1-5] MIG process is highly nonlinear and the shape of line produced by it is affected by four parameters feed rate, travel speed, welding current and arc voltage. The relation between each parameter and the shape is not exactly identified but known approximately as proportionality[6] so modeling them is important for enhancing usages of the process. Neural network is the best method to model such process [7-9] so it was used in this research after developing suitable script for each shape property.

Lately, Artificial Intelligence (Al) has emerged as a powerful tool thus opening the door to the solution of more challenging problems such as those found in metal deposition using MIG. ANN a class of Al represents a growing new technology as indicated by their ability to learn complex nonlinear relationships and by the wide variety of applications.

ANNs simulate the human intelligence and are characterized by a robust generalization and error tolerance found in human beings. They can discern patterns and relationships that are beyond the capabilities of the numerical methods such as regression and pattern recognition. The biological brain is the nerve cell or neuron that acts as a simplified numerical processor. In fact, the brain contains billions of such neurons, all heavily interconnected and operating in parallel. In biology, the memory is believed to be stored in the strength of the connections between layers. In ANN, such interconnections are known as weight. Such interconnects connect neurons in different layers.

Usually, ANNs can be defined as an interconnected array of neurons. A popular model is the multilayered one made of several layers. The first one receives the input vector whose elements are weighted to form at the hidden layers a weighted sum that is altered by a function to calculate the output of each hidden neuron. A linear combination of the hidden layer neural outputs is used to generate the outputs. ANNs must pass two steps training and testing. During training, weights are modified according to the difference between the output and the targets. The network is retrained, until converge is obtained. The learning algorithm uses back propagation. The error, which is the difference of the ANN output and the target, is calculated and propagated backwards from the output to hidden layer, and then to the input. The process is repeated until the difference between all output nodes and all targets become within some accepted tolerance.

\section{TRAINING METHOD}

In supervised learning for each input, the correct output is known and this information 
is given to the neural network. This is supervised learning since the neural network learns under supervision like student and teacher. The back propagation model is such an example, assuming an existence of a teacher who knows what correct patterns are. In the back propagation model, the actual output from the neural network is compared with the correct one, and the weights are adjusted to reduce the difference. Maintaining the Integrity of the Specifications

Outline of the learning (training) algorithm:

Outer loop: Repeat steps so the neural network can consecutively map all patterns sufficiently.

Inner loop: For each pattern, repeat the following Steps 1 to 3 until the output Vector $y$ is close enough to the target vector $t$ for the given input vector $x$.

Step1: Enter $x$ to the input layer of neural network.

Step2: Feed forward. Go through the neural network, from the input to hidden Layers, then from the hidden to output layers, and get output vector $y$.

Step3: Backward propagation of error corrections. Compare y with $t$. If $y$ is equal or close enough to then go back to the beginning of the Outer loop. Otherwise, back propagate through the neural network and adjust the weights so that the next $y$ is closer to $t$, then go back to the beginning of the Inner loop.

There are two commonly used criteria to terminate iterations. The first is the absolute error between the output $y$ and the target $t$ whereas the second is the (mean square error) MSE.

The Outer loop iteration is called an epoch. An epoch is one cycle through the entire set of patterns under consideration Note that to terminate the outer loop (i.e., the entire algorithm); the neural network must be able to produce the target vector for any input vector.

To prevent repeating the computation, ANNs are trained off-line then used to predict metal deposition process using MIG.

\section{METHODOLOGY AND DISCUSSION}

Metal $30 \mathrm{~mm}$ liens were deposited on st37 strips of $100 \times 30 \times 4 \mathrm{~mm}$ using KUKA robot and Fronious welding machine. Ranges of the parameters used were selected according to AWS, Miller Company's android application and Fronious ios application welding parameters are as shown in Table.1.

Table 1. MIG welding parameters.

\begin{tabular}{|l|c|}
\hline \multicolumn{1}{|c|}{ Wire type } & ER70S-6 \\
\hline Current, [Amp] & $180: 190$ \\
\hline Voltage, [Volts] & $24: 25$ \\
\hline CTWD, [mm] & 12.7 \\
\hline Travel speed, [cm/min] & $10: 125$ \\
\hline Feed rate, [IPM] & $360: 380$ \\
\hline Gas Mixture & Argon $75 \%$ and CO2 25\% \\
\hline
\end{tabular}


Feed rate was supposed to be one of the controlled parameters but the welding machine practically changes it according to material, work piece thickness, volt and current. The selected wire type is recommended by AWS for the requirement of higher deoxidization which may occur when welding rusty or non-perfectly clean steel pieces. Ratios of gas mixture are according to previous researches and were preferred to cope with Egyptian gas suppliers capabilities.

A Taguchi design was performed taking 5 levels for each controllable parameter. Only 3 parameters were considered controllable they are voltage, current and travel speed while feed rate was to be observed and other parameters are kept constant. Taguchi designed 25 experiments. Ranges and levels are shown in Table.2.

Table 2. Parameters, ranges and levels.

\begin{tabular}{|l|c|c|}
\hline \multicolumn{1}{|c|}{ Parameter } & Range & Levels \\
\hline Voltage, [Volt] & $24: 25$ & $24,24.3,24.6,24.9,25$ \\
\hline Current, [Amp] & $180: 190$ & $180,183,186,189,190$ \\
\hline Travel speed, [m/sec] & $0.003: 0.025$ & $\begin{array}{c}0.003,0.008,0.013, \\
0.018,0.025\end{array}$ \\
\hline
\end{tabular}

Each input set of the 25 was repeated 3 times then each specimen width was measured over the deposited line then each specimen was cut cross sectional in order to measure height and penetration. Vernier caliper with resolution of $0.02 \mathrm{~mm}$ was used to take these measurements. For each specimen as the one shown in Fig. 1 5 width measurements were taken along the line equally distanced starting and ending $5 \mathrm{~mm}$ from the start and end of the line. Then a quartile test was performed because some specimens were suspected to be unreasonably changing in width along the line. Only 8 measurements from different 5 specimens were outliers so they were excluded from the data set. The mean width and standard deviation of each specimen was calculated in order not to miss the irregularity of the line width in some specimens.

Then each specimen was cut horizontally at $10 \mathrm{~mm}$ from the line start to measure height and penetration. An etching solution of $2 \%$ nitric acid and $98 \%$ ethanol was used to recognize the bead and take measurements of penetration. First the height was measured then the whole bead length was measured the difference between them is the penetration this method was used to decrease any possible error in measurements.

Data was used to make neural networks representing the relation between the parameters used for depositing the lines and the shape of the line. The shape here means line width (represented by mean values and standard deviation), penetration and height. Many neural networks were designed to model the collected data.

First group used all inputs and outputs for networks training including the observation excluding two experiments for further testing of the resulting networks. Best of Networks which passed the two experiments test with tolerance of $\pm 2 \%$ maximum showed regression ranging between 76 to $90 \%$ and a MSE (mean square error) ranging between 0.23 and 1.13 . These results were not satisfying so the model was 
divided into networks each one resembling the relation between all inputs and one output in order to decrease confusion of the network.

\section{A. Width}

Some networks were designed to model the relation between all inputs and the width. These networks showed regression more than 99 and MSE ranging between 0.16 and 0.32 . And all these networks passed the $\pm 2 \%$ test. It consisted of 2 hidden layers containing 5 and 4 neurons respectively with "tansig" functions and an output layer of linear function. Trainlm function gave the best result for width.

\section{B. Penetration}

The networks modeling the relation between all inputs and penetration did not pass the $\pm 2 \%$ test at all so the tolerance was increased to $\pm 5 \%$ but it was not sufficient so it was increased to $\pm 10 \%$ then some networks passed the test showing regression ranging from 68 to 86 and MSE ranged from 0.06 to 0.23 . It consisted of 5 layers containing 10, 15, 15, 25 and 30 respectively with "tansig" functions and an output layer of linear function. Traingd function gave the best result for penetration.

\section{Height}

The best networks made to model the height showed regression of 68 to 92 and MSE of 0.03 to 0.08 . It consisted of 1 hidden layer containing 7 neurons of "tansig" function and an output layer with function of "purelin". For better results it was suggested to make other networks for height and penetration related to the effective inputs known from previous researches. None of the developed networks with single input single output showed reasonable results most of them showed over fitting and negative regression, so the multi input multi output are to be accepted. Traingd function gave the best result for height.

For width the sixth network was found to be the best network among those passed the $\pm 2 \%$ test as it showed the highest regression with least MSE (mean square error) without over fitting as shown in Figs 2 and 3.

This network resembles the relation between the 3 inputs (voltage welding current and travel speed) and the width represented by the mean and standard deviation because there was large variation in the width along weld line in some specimens.

For penetration the best network was also the sixth it showed the highest regression with least MSE without over fitting. Its regression and MSE values are shown in Figs. 4 and 5 . This network resembles the relation between the 3 inputs (voltage welding current and travel speed) and the penetration.

It was thought to make single input single output with guidance of appendix 4 which summarizes information from the AMS [6] in order to get better networks so for penetration current was chosen as input but results were shockingly unreasonable but answer for that was found in [10] as it found penetration to be affected by voltage also but not with the same significance as current. Even travel speed was found to be effective on penetration in this research because if it was relatively low. 
It can cause metal accumulation and decrease penetration. That makes penetration affected by the three chosen parameters not with the same impact of course but affected anyhow. The paper approximated the ratio between current effect and effect of both arc voltage and welding speed by 2.5 times.

For height the first network was thought to be the most representative one among networks which passed the simulation test but this time with error tolerance of $\pm 5 \%$ of the measured samples ranges measured for each input data set of the two samples excluded for test purpose.it was selected although its regression was not so high as shown in Fig. 6 because its MSE was low enough as shown in Fig.7 and there was not over fitting.

\section{CONCLUSION}

1. The three input parameters of Voltage Current and travel speed were effective in the three outputs of width, penetration and height and excluding any one of them was to be a big mistake.

2. Testing neural networks with untrained experiments is essential as regression graphs and MSE (mean square error) are not enough for judging networks.

3. For width the best network was consisted of 2 hidden layers containing 5 and 4 neurons respectively with "tansig" functions and an output layer of linear function.

4. For height the best network was consisted of 1 hidden layer containing 7 neurons of "tansig" function and an output layer with function of "purelin".

5. For penetration the best network was consisted of 5 layers containing $10,15,15$, 25 and 30 respectively with "tansig" functions and an output layer of linear function.

\section{FUTURE WORK}

- Study the effect of the three parameters on hardness of the deposited metal line.

- Determine the optimal parameters for depositing metal line with best shape.

- Determine the optimal parameters for depositing metal line without affecting hardness too much.

\section{ACKNOWLEDGMENT}

Thanks for T.E.S technological and electrical systems company and its' chairman for support and supplying equipment for this research.

\section{REFERENCES}

[1] Kah, P., Latifi, Hamidreza, Suoranta, Raimo, Martikainen, Jukka, Pirinen, Markku, Usability of arc types in industrial welding. International Journal of Mechanical and Materials Engineering, 9(1) (2014).

[2] Santosh, "Robot Based 3D Welding For Jet Engine Blade Repair And Rapid Prototyping Of Small Components", Master of Science, Dept. of Mech. and Manuf. Engineering, University of Manitoba Winnipeg, Manitoba, Canada. 
[3] Cruz, J.G., E.M. Torres, and S.C. Absi Alfaro, A methodology for modeling and control of weld bead width in the GMAW process. Journal of the Brazilian Society of Mechanical Sciences and Engineering, 37(5): p. 1529-1541.R. Nicole (2015).

[4] Kim, J.-S., I.-J. Kim, and Y.-G. Kim, Optimization of weld pitch on overlay welding using mathematical method. International Journal of Precision Engineering and Manufacturing, 15(6): p. 1117-1124 (2014).

[5] Xiong, J., et al., Forecasting process parameters for GMAW-based rapid manufacturing using closed-loop iteration based on neural network. The International Journal of Advanced Manufacturing Technology, 69(1-4): p. 743751 (2013).

[6] ASM Handbook - Vol 06 - Welding, Brazing, and Soldering (2873s).pdf.

[7] LinWu, J.X.G.Z.J.H., Bead geometry prediction for robotic GMAW-based rapidmanufacturing through a neural network and a second-orderregression analysis. Intell Manuf, p. 25 (2014).

[8] Malviya, R. and D.K. Pratihar, Tuning of neural networks using particle swarm optimization to model MIG welding process. Swarm and Evolutionary Computation, 1(4): p. 223-235 (2011).

[9] Ates, H., Prediction of gas metal arc welding parameters based on artificial neural networks. Materials \& Design, 28(7): p. 2015-2023 (2007).

[10] Karadeniz, E., U. Ozsarac, and C. Yildiz, The effect of process parameters on penetration in gas metal arc welding processes. Materials \& Design, 28(2): p. 649-656 (2007). 


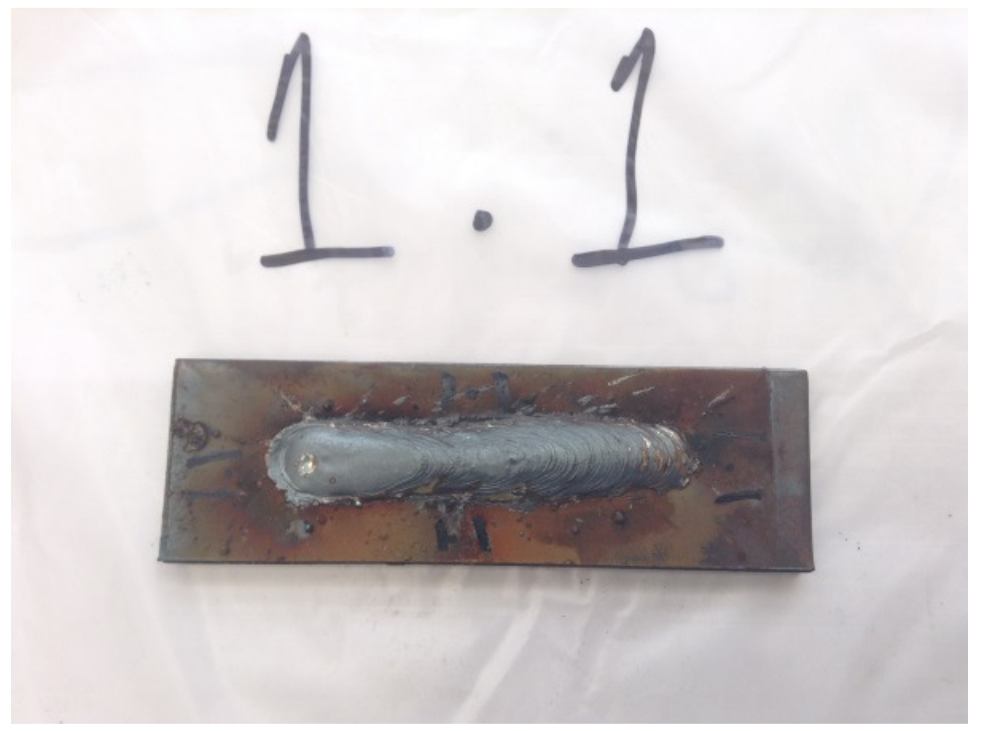

Fig.1. Weld line specimen.
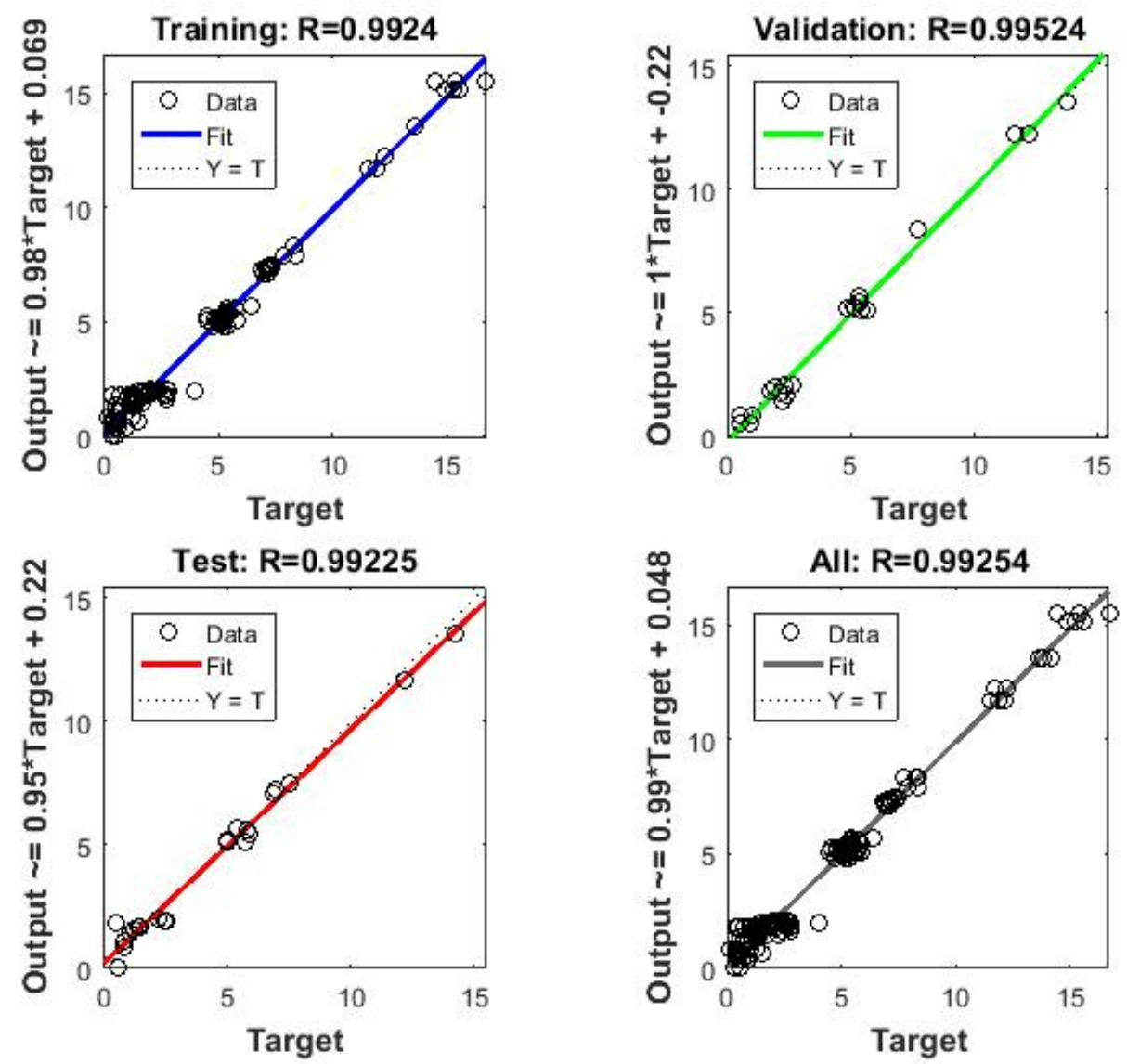

Fig.2. Best width network regression. 


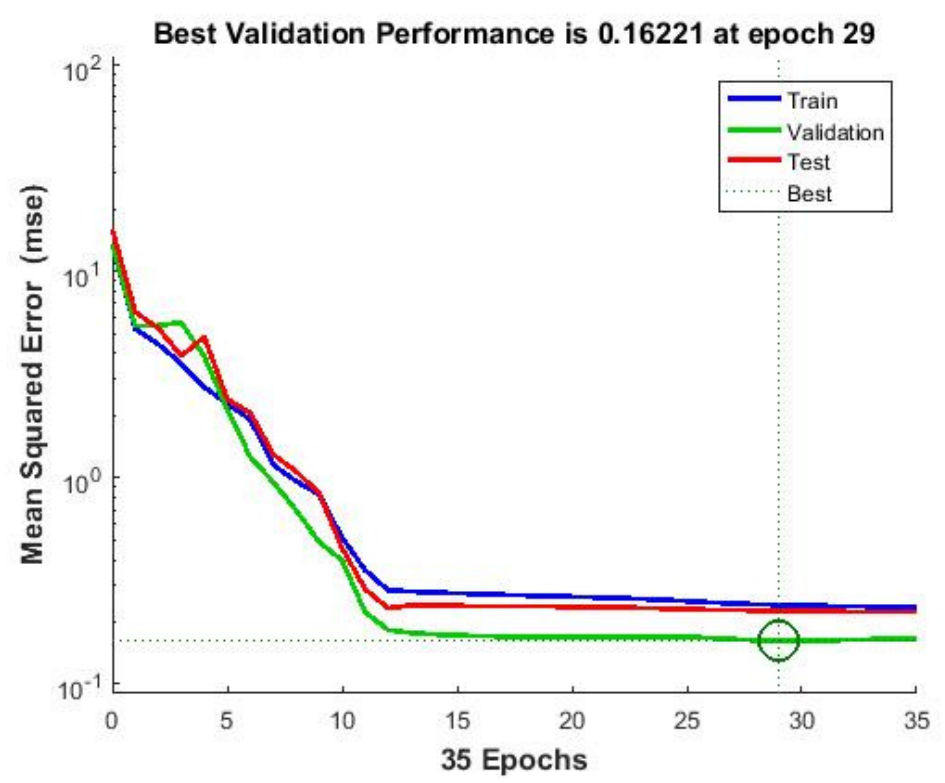

Fig.3. Best width network MSE
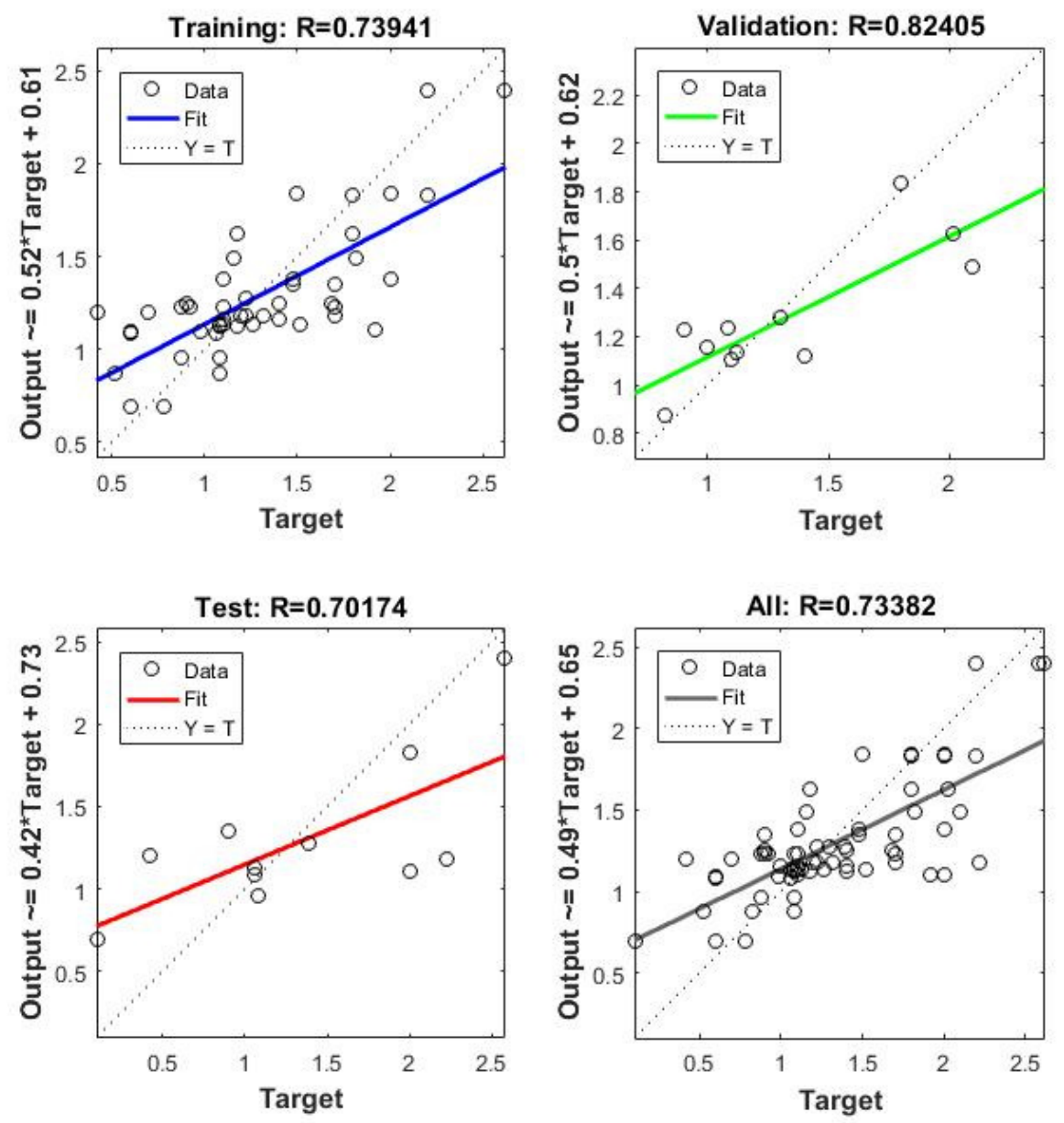

Fig. 4. Best penetration network regression. 


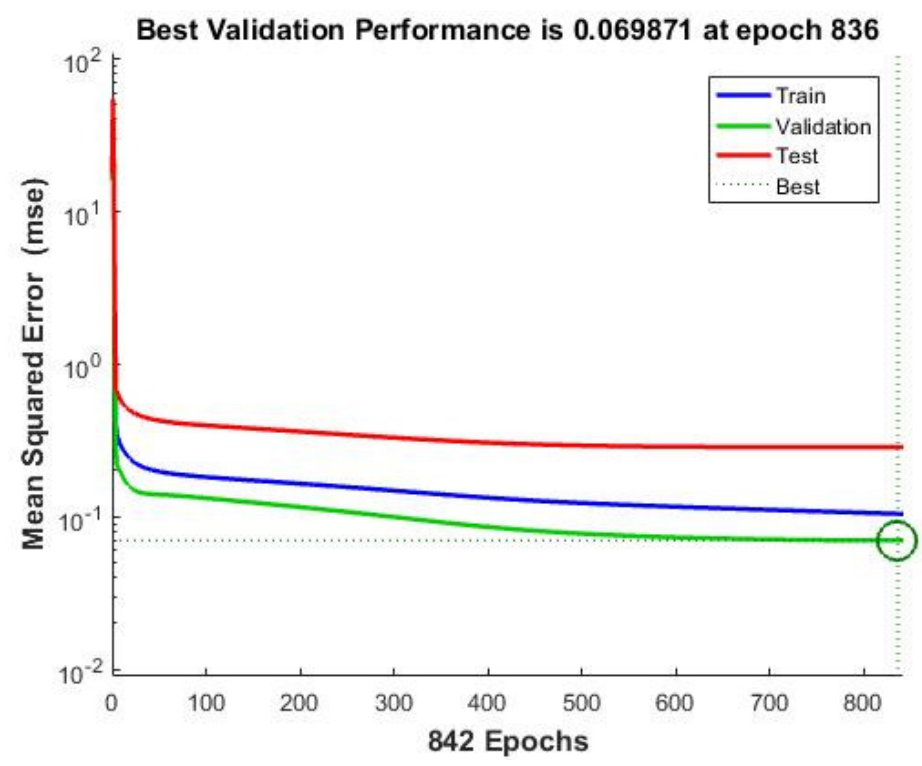

Fig. 5. Best penetration network MSE.
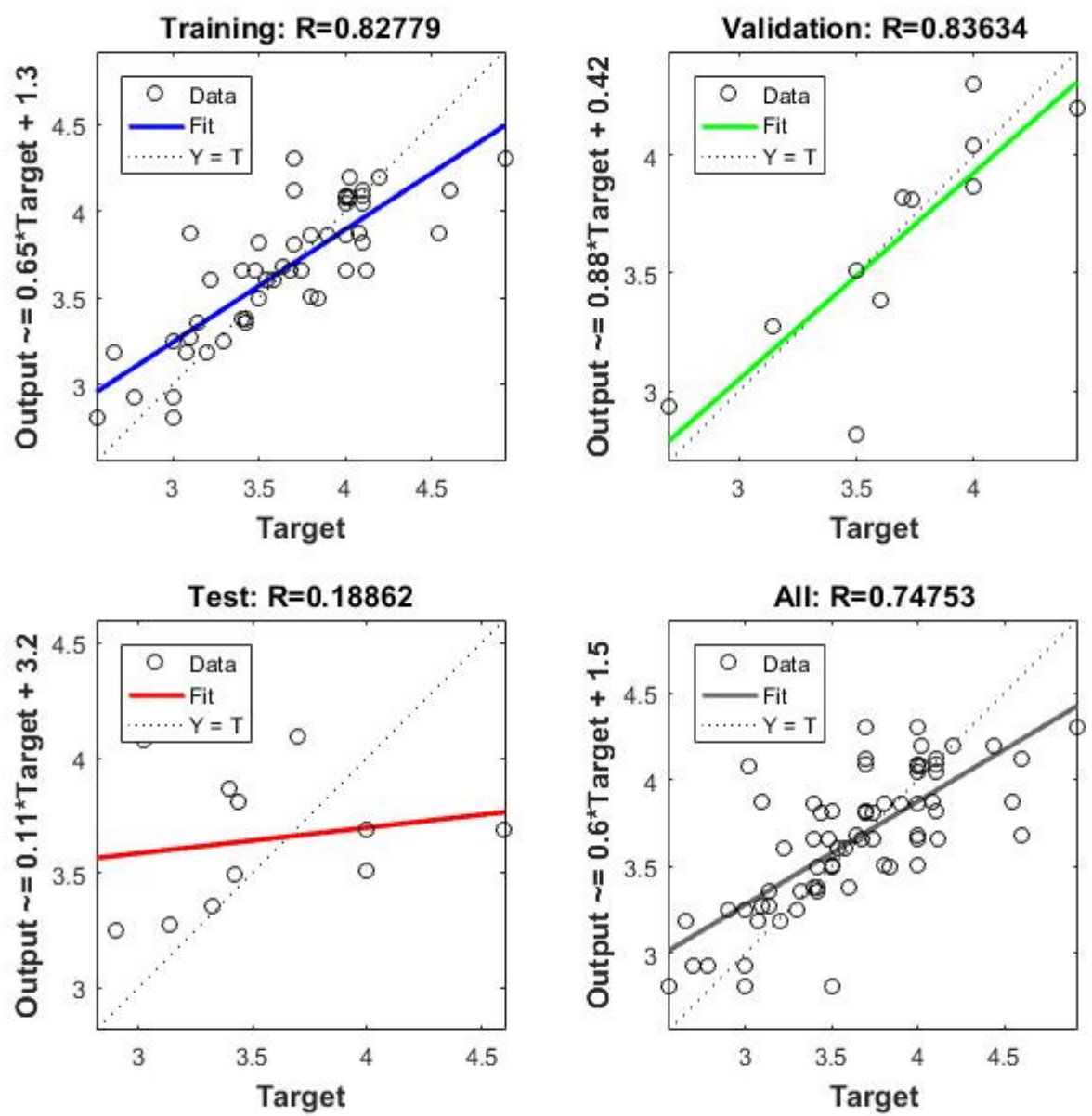

Fig. 6. Best height network regression. 


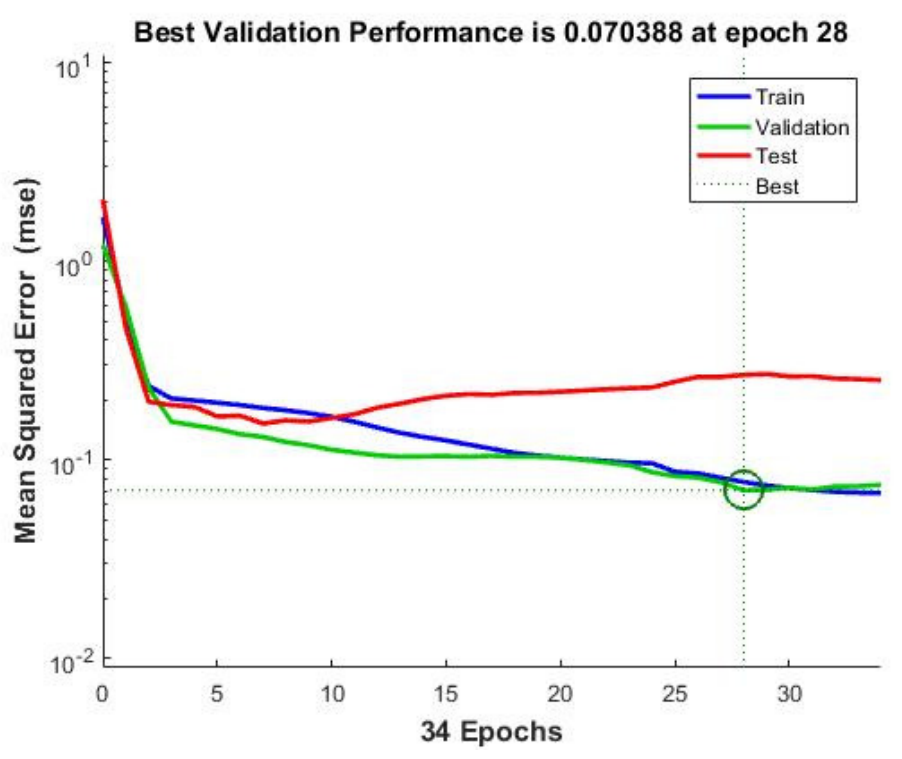

Fig. 7. Best height network MSE. 